\title{
IV. Symposium of Young Researchers on Pharmaceutical \\ Technology, Biotechnology and Regulatory Science
}

January 19-21, 2022 - Szeged, Hungary

DOI: $10.14232 /$ syrptbrs.2022.59

\section{Pharmaceutical study of essential oil-loaded liposomal formulations}

Reza Semnani Jazani, Zsófia Németh, Dorina Gabriella Dobó, Ildikó Csóka

University of Szeged, Faculty of Pharmacy, Institute of Pharmaceutical Technology and Regulatory Affairs, Szeged, Hungary

Liposomes are lipohydrophilic nanocarriers used in drug delivery and exhibited substantial clinical successes. Essential oils (EO) are lipophilic chemicals with several medical and physiological effects. Studies suggest that not only liposomes are promising carriers for EOs and increase their stability but also EOs have effects on the liposomal structure. There is still a lack of data on this field; thus, the topic requires more attention for better investigation of the possible evaluation (1).

Accordingly, the goal of this work was to study the effects of different kinds and ratios of EOs on liposomal formulations.

Liposomes were made from phosphatidylcholine, cholesterol (60:40 weight ratio), and a diverse range of ratios of EOs (5-200 percentage of the whole lipid mass concentration), using the thin-film hydration preparation method (2). The vesicles were characterized by a zeta-sizer via the dynamic light scattering method. The zeta potential and vesicle size values were investigated, and furthermore, the possible correlation between various EOs with different concentrations was identified in this study.

After adding a certain ratio of EOs to the formulations, those have become polydisperse (higher polydispersity index than 0.3) and bigger than $200 \mathrm{~nm}$ size was measured. Zeta potential values tend to be negative, and a correlation between them was detected.

The impact of EOs on liposomal formulation has been shown. These data may be used for liposomal optimization and simultaneously also to increase the bioavailability of EOs.

References

1. Sao Pedro A. Formatex (Ed. Méndez-Vilas A.) 1364-1374 (2013)

2. Zhang H. Methods Mol Biol. 1522, 17-22 (2017) 